\title{
Bounds on the Number of Iterations for Turbo-Like Code Ensembles over the Binary Erasure Channel
}

\author{
Igal Sason Gil Wiechman \\ Department of Electrical Engineering \\ Technion, Haifa 32000, Israel \\ \{sason@ee, igillw@tx\}.technion.ac.il
}

\begin{abstract}
We derive simple lower bounds on the number of iterations which are required to communicate over the binary erasure channel when graph-based code ensembles are used in conjunction with an iterative message-passing decoder. These bounds refer to the asymptotic case where we let the block length tend to infinity, and the bounds apply to general ensembles of lowdensity parity-check (LDPC) codes, irregular repeat-accumulate (IRA) and accumulate-repeat-accumulate (ARA) codes. It is demonstrated that, under a mild condition, the number of iterations required for successful decoding scales at least like the inverse of the gap (in rate) to capacity.
\end{abstract}

\section{INTRODUCTION}

The number of iterations, used by an iterative messagepassing decoder for graph-based codes, forms an important factor in the evaluation of the decoding delay and complexity. Another important factor which affects the complexity of such decoders is related to the graphical complexity of the Tanner graphs used to represent these codes (this graphical complexity is strongly related to the decoding complexity per iteration of message-passing decoders).

The extrinsic information transfer (EXIT) charts, pioneered by Stephan ten Brink [15], form a powerful tool for an efficient design of codes defined on graphs by tracing the convergence behavior of their iterative decoders. For the binary erasure channel (BEC), the EXIT charts coincide with the density evolution analysis, and both are simplified in this case to a one-dimensional analysis.

A numerical approach for the joint optimization of the design rate and decoding complexity of LDPC code ensembles was provided in [2]. For simplifying this optimization problem, a suitable approximation of the number of iterations was used in order to convert the original problem into a convex optimization problem. This approach applies to the case where the transmission takes place over a memoryless binary-input output-symmetric (MBIOS) channel, and we let the block length tend to infinity. An alternative approach, used to approximate the number of iterations for LDPC code ensembles operating over the BEC is addressed in [6].

An analytical approach used to characterize the asymptotic complexity of iterative decoders, under the assumption where the block length tends to infinity, was suggested by Khandekar and McEliece [4]. The questions raised in [4] and the following conjectures are related to the tradeoff between the asymptotic achievable rates and the complexity under iterative message-passing decoding. It was conjectured in [4] that for a large class of channels, if the design rate of a suitably designed ensemble forms a fraction $1-\varepsilon$ of the channel capacity, then the decoding complexity (per information bit) scales like $\frac{1}{\varepsilon} \log \frac{1}{\varepsilon}$. The logarithmic term in this expression was attributed to the graphical complexity (i.e., the decoding complexity per iteration), and the number of iterations was conjectured to scale like $\frac{1}{\varepsilon}$. There is one exception: For the BEC, the complexity under the iterative message-passing decoding algorithm behaves like $\ln \frac{1}{\varepsilon}$ (see [5], [11], [12] and [14]). This is true since the absolute reliability provided by the BEC allows every edge in the graph to be used only once during the iterative decoding. Hence, for the BEC, the number of iterations performed by the decoder serves mainly to measure the delay in the decoding process, while the decoding complexity is closely related to the complexity of the Tanner graph which is chosen to represent the code.

This paper provides simple lower bounds on the number of iterations which are required for successful message-passing decoding of graph-based code ensembles. The transmission of these ensembles is assumed to take place over the BEC, and the bounds refer to the asymptotic case where the block length tends to infinity. The simplicity of the bounds derived in this paper stems from the fact that they are easily evaluated and are expressed in terms of some basic parameters of the considered ensemble. These bounds demonstrate that the number of iterations which are required for successful messagepassing decoding scales at least like the inverse of the gap (in rate) to capacity, provided that the fraction of degree- 2 variable nodes of these turbo-like ensembles does not vanish (hence, the number of iterations becomes unbounded as the gap to capacity vanishes). The behavior of these lower bounds matches well with the experimental results and the conjectures on the number of iterations and complexity, as provided by Khandekar and McEliece [4].

This paper is structured as follows: Section II presents some preliminary background, definitions and notation, Section III introduces the main results of this work and discusses some of their implications, outlines for the proofs of the main statements are provided in Section IV. Finally, Section V summarizes this paper. Due to space limitations, the full proofs, as well as several discussions, are omitted; the interested reader is referred to the full paper version which is available on the arxiv [13]. 


\section{PRELIMINARIES}

This section provides preliminary background and introduces notation for the rest of this paper.

\section{A. Accumulate-Repeat-Accumulate Codes}

Accumulate-repeat-accumulate (ARA) codes form an attractive coding scheme of turbo-like codes due to the simplicity of their encoding and decoding (where both scale linearly with the block length), and due to their remarkable performance under iterative decoding [1].

Ensembles of irregular and systematic ARA codes, which asymptotically achieve the capacity of the BEC with bounded graphical complexity, are presented in [9]. This bounded complexity result stays in contrast to LDPC code ensembles, which have been shown to require unbounded graphical complexity in order to approach channel capacity, even under maximumlikelihood decoding (see [11]). The setting and notation used to characterize ensembles of irregular and systematic ARA codes and their iterative decoders are introduced in [9, Section II] (due to space limitations, we refer the reader to [9]).

\section{B. Big-O notation}

The terms $O, \Omega$ and $\Theta$ are widely used in computer science to describe asymptotic relationships between functions. In our context, we refer to the gap (in rate) to capacity, denoted by $\varepsilon$, and discuss in particular the case where $0 \leq \varepsilon \ll 1$ (i.e., sequences of capacity-approaching ensembles). Accordingly, we define

- $f(\varepsilon)=O(g(\varepsilon))$ means that there are positive constants $c$ and $\delta$, such that $0 \leq f(\varepsilon) \leq c g(\varepsilon)$ for all $0 \leq \varepsilon \leq \delta$.

- $f(\varepsilon)=\Omega(g(\varepsilon))$ means that there are positive constants $c$ and $\delta$, such that $0 \leq c g(\varepsilon) \leq f(\varepsilon)$ for all $0 \leq \varepsilon \leq \delta$.

- $f(\varepsilon)=\Theta(g(\varepsilon))$ means that there are positive constants $c_{1}, c_{2}$ and $\delta$, such that $0 \leq c_{1} g(\varepsilon) \leq f(\varepsilon) \leq c_{2} g(\varepsilon)$ for all $0 \leq \varepsilon \leq \delta$.

For all the above definitions, the values of $c, c_{1}, c_{2}$ and $\delta$ must be fixed for the function $f$ and should not depend on $\varepsilon$.

\section{MAIN RESULTS}

In this section, we present lower bounds on the required number of iterations used by a message-passing decoder for code ensembles defined on graphs. The communication is assumed to take place over a BEC, and we consider the asymptotic case where the block length of these code ensembles tends to infinity.

Definition 3.1: Let $\left\{\mathcal{C}_{m}\right\}_{m \in \mathbb{N}}$ be a sequence of code ensembles. Assume a common block length $\left(n_{m}\right)$ of the codes in $\mathcal{C}_{m}$ which tends to infinity as $m$ grows. Let the transmission of this sequence take place over a BEC with capacity $C$. The sequence $\left\{\mathcal{C}_{m}\right\}$ is said to achieve a fraction $1-\varepsilon$ of the channel capacity under some given decoding algorithm if the asymptotic rate of the codes in $\mathcal{C}_{m}$ satisfies $R \geq(1-\varepsilon) C$ and the achievable bit erasure probability under the considered algorithm vanishes as $m$ becomes large.

In the continuation, we consider a standard iterative messagepassing decoder for the BEC, and address the number of iterations which are required in terms of the achievable fraction of the channel capacity under this decoding algorithm.

Theorem 3.1: [Lower bound on the number of iterations for LDPC code ensembles transmitted over the BEC]. Let $\left\{\left(n_{m}, \lambda, \rho\right)\right\}_{m \in \mathbb{N}}$ be a sequence of LDPC code ensembles whose transmission takes place over a BEC with erasure probability $p$. Assume that this sequence achieves a fraction $1-\varepsilon$ of the channel capacity under message-passing decoding. Let $L_{2}=L_{2}(\varepsilon)$ be the fraction of variable nodes of degree 2 for this sequence. In the asymptotic case where the block length tends to infinity, let $l=l\left(\varepsilon, p, P_{\mathrm{b}}\right)$ denote the number of iterations which are required to achieve an average bit erasure probability $P_{\mathrm{b}}$ over the ensemble. Under the mild condition that $P_{\mathrm{b}}<p L_{2}(\varepsilon)$, the required number of iterations satisfies the lower bound

$$
l\left(\varepsilon, p, P_{\mathrm{b}}\right) \geq \frac{2}{1-p}\left(\sqrt{p L_{2}(\varepsilon)}-\sqrt{P_{\mathrm{b}}}\right)^{2} \frac{1}{\varepsilon} .
$$

Discussion 3.1: [On the dependence of the bounds on the fraction of degree-2 variable nodes] The lower bound on the number of iterations in Theorem 3.1 becomes trivial when the fraction of variable nodes of degree 2 vanishes. In fact, for various sequences of capacity approaching LDPC code ensembles known to date (see [5], [7], [14]), the statement in [10, Lemma 5] implies that the fraction of degree-2 variable nodes tends to $\frac{1}{2}$ irrespectively of the erasure probability of the BEC, as can be verified directly for these code ensembles.

In the asymptotic case where the block length tends to infinity and the transmission takes place over the BEC, suitable constructions of capacity-achieving systematic ARA ensembles enable a fundamentally improved tradeoff between their graphical complexity and their achievable gap (in rate) to capacity under iterative decoding, as compared to LDPC code ensembles (see [9],[11]). This raises the question whether the number of iterations required to achieve a desired bit erasure probability under iterative decoding, can be reduced by using systematic ARA ensembles. The following theorem shows that similarly to the result for LDPC code ensembles (see Theorem 3.1), the required number of iterations for systematic ARA codes scales at least like the inverse of the gap to capacity.

Theorem 3.2: [Lower bound on the number of iterations for systematic ARA ensembles transmitted over the BEC]. Let $\left\{\left(n_{m}, \lambda, \rho\right)\right\}_{m \in \mathbb{N}}$ be a sequence of systematic ARA ensembles whose transmission takes place over a BEC with erasure probability $p$. Assume that this sequence achieves a fraction $1-\varepsilon$ of the channel capacity under message-passing decoding. Let $L_{2}=L_{2}(\varepsilon)$ be the fraction of 'punctured bit' nodes of degree 2 for this sequence (see [9, Fig. 2] where the two edges related to the accumulator are not taken into account). Let the block length tend to infinity, and let $l=l\left(\varepsilon, p, P_{\mathrm{b}}\right)$ denote the required number of iterations to achieve an average bit erasure probability $P_{\mathrm{b}}$ of the systematic bits. Under the mild condition that $1-\sqrt{1-\frac{P_{\mathrm{b}}}{p}}<p L_{2}(\varepsilon)$, 
this number of iterations satisfies the lower bound

$l\left(\varepsilon, p, P_{\mathrm{b}}\right) \geq 2 p(1-\varepsilon)\left(\sqrt{p L_{2}(\varepsilon)}-\sqrt{1-\sqrt{1-\frac{P_{\mathrm{b}}}{p}}}\right)^{2} \frac{1}{\varepsilon}$.

The following theorem, which refers to irregular repeataccumulate (IRA) code ensembles, is proved in a conceptually similar way to the proof of Theorem 3.2.

Theorem 3.3: [Lower bound on the number of iterations for IRA code ensembles transmitted over the BEC]. Let $\left\{\left(n_{m}, \lambda, \rho\right)\right\}_{m \in \mathbb{N}}$ be a sequence of (systematic or nonsystematic) IRA code ensembles whose transmission takes place over a BEC with erasure probability $p$. Assume that this sequence achieves a fraction $1-\varepsilon$ of the channel capacity under message-passing decoding. Let $L_{2}=L_{2}(\varepsilon)$ be the fraction of 'information bit' nodes of degree 2 for this sequence. In the asymptotic case where the block length tends to infinity, let $l=l\left(\varepsilon, p, P_{\mathrm{b}}\right)$ designate the number of iterations required to achieve an average bit erasure probability $P_{\mathrm{b}}$ of the information bits. For systematic codes, if $P_{\mathrm{b}}<p L_{2}(\varepsilon)$, then the number of iterations satisfies the lower bound

$$
l\left(\varepsilon, p, P_{\mathrm{b}}\right) \geq 2(1-\varepsilon)\left(\sqrt{p L_{2}(\varepsilon)}-\sqrt{P_{\mathrm{b}}}\right)^{2} \frac{1}{\varepsilon} .
$$

For non-systematic codes, if $P_{\mathrm{b}}<L_{2}(\varepsilon)$, then

$$
l\left(\varepsilon, p, P_{\mathrm{b}}\right) \geq 2(1-\varepsilon)\left(\sqrt{L_{2}(\varepsilon)}-\sqrt{P_{\mathrm{b}}}\right)^{2} \frac{1}{\varepsilon} .
$$

\section{Outline for the Proofs of the Main Results}

\section{A. Outline for the Proof of Theorem 3.1}

Let $\left\{x^{(l)}\right\}_{l \in \mathbb{N}}$ designate the expected fraction of erasures in messages from the variable nodes to the check nodes at the $l$ 'th iteration of the message-passing decoder (where we start counting at $l=0$ ). From density evolution, in the asymptotic case where the block length tends to infinity, the average bit erasure probability after the $l$ 'th decoding iteration is given by

$$
P_{\mathrm{b}}^{(l)}=p L\left(1-\rho\left(1-x^{(l)}\right)\right)
$$

where $p$ is the erasure probability of the BEC and $L$ designates the left degree distribution of the ensemble from the node perspective. Density evolution also implies that an average bit erasure probability of $P_{\mathrm{b}}$ is attainable under iterative decoding if and only if

$$
c(x)<v(x), \quad \forall x \in\left(x^{*}, p\right]
$$

where the functions $c$ and $v$ are defined as

$$
c(x) \triangleq 1-\rho(1-x), \quad v(x) \triangleq \begin{cases}\lambda^{-1}\left(\frac{x}{p}\right) & 0 \leq x \leq p \\ 1 & p<x \leq 1\end{cases}
$$

and $x^{*}$ is the unique solution of $P_{\mathrm{b}}=p L\left(1-\rho\left(1-x^{*}\right)\right)$. Since we assume that vanishing bit erasure probability is achievable under message-passing decoding, it follows that $c(x)<v(x)$ for all $x \in(0, p]$. Figure 1 shows a plot of the functions $c(x)$ and $v(x)$ for an ensemble of LDPC codes which achieves vanishing bit erasure probability under iterative decoding as the block length tends to infinity. The horizontal and vertical lines, labeled $\left\{h_{l}\right\}_{l \in \mathbb{N}}$ and $\left\{v_{l}\right\}_{l \in \mathbb{N}}$, respectively, are used to track the expected fraction of erased messages from the variable nodes to the check nodes at each iteration of the message-passing decoding algorithm. The expected fraction of erased left to right messages in the l'th decoding iteration (where we start counting at zero) is equal to the $x$ value at the left tip of the horizontal line $h_{l}$. The right-angled triangles shaded in gray will be used later in the proof.

The first step in the proof of Theorem 3.1 is calculating the area bounded by the curves $c(x)$ and $v(x)$. This is done in the following lemma which is based on the area theorem for the BEC [3].

Lemma 4.1:

$$
\int_{0}^{1}(v(x)-c(x)) \mathrm{d} x=\frac{C-R}{a_{\mathrm{L}}}
$$

where $C=1-p$ is the capacity of the BEC, $R$ is the design rate of the ensemble, and $a_{\mathrm{L}}$ is its average left degree.

Let us consider the two sets of right-angled triangles shown in two shades of gray in Figure 1. The triangles shaded in dark gray are defined so that one of the legs of triangle number $i$ (counting from right to left and starting at zero) is the vertical line $v_{i}$, and the slope of the hypotenuse is equal to $c^{\prime}(0)=$ $\rho^{\prime}(1)$. The area of the $i$ 'th triangle in this set is

$$
A_{i}=\frac{1}{2}\left|v_{i}\right|\left(\frac{\left|v_{i}\right|}{\rho^{\prime}(1)}\right)=\frac{\left|v_{i}\right|^{2}}{2 \rho^{\prime}(1)}
$$

where $\left|v_{i}\right|$ is the length of $v_{i}$. The triangles shaded in light gray are defined so that one of the legs of triangle number $i$ (again, counting from the right and starting at zero) is the vertical line $v_{i}$ and the slope of the hypotenuse is given by

$$
v^{\prime}(0)=\frac{1}{p}\left(\lambda^{-1}\right)^{\prime}(0)=\frac{1}{p \lambda^{\prime}(0)}=\frac{1}{p \lambda_{2}}
$$

where the second equality follows since $\lambda(0)=0$. The area of the $i$ 'th triangle in this second set of triangles is given by

$$
B_{i}=\frac{1}{2}\left|v_{i}\right|\left(\left|v_{i}\right| p \lambda_{2}\right)=\frac{p \lambda_{2}\left|v_{i}\right|^{2}}{2} .
$$

Since $c(x)$ and $v(x)$ are monotonically increasing with $x$ and concave, both sets of triangles form a subset of the domain bounded by the curves of $c(x)$ and $v(x)$. By their definitions, the $i$ 'th dark triangle is on the right of $v_{i}$, and the $i$ 'th light triangle lies to the left of $v_{i}$; therefore, the triangles do not overlap. Combining (7), (8) and the fact that the triangles do not overlap, and applying Lemma 4.1, we get

$$
\frac{C-R}{a_{\mathrm{L}}} \geq \frac{1}{2}\left(\frac{1}{\rho^{\prime}(1)}+p \lambda_{2}\right) \sum_{i=0}^{l-1}\left|v_{i}\right|^{2}
$$

where $l$ is an arbitrary natural number. Since we assume that the bit erasure probability vanishes under message-passing decoding, the stability condition implies that $\frac{1}{\rho^{\prime}(1)} \geq p \lambda_{2}$. 


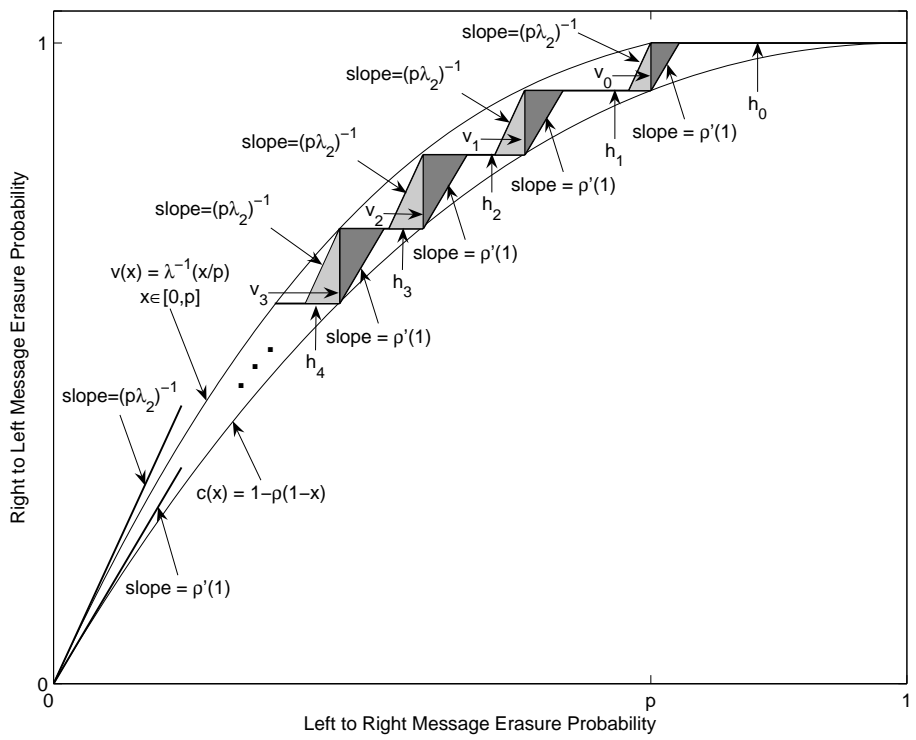

Fig. 1. Plot of the functions $c(x)$ and $v(x)$ for an ensemble of LDPC codes which achieves vanishing bit erasure probability under iterative message-passing decoding when communicated over a BEC whose erasure probability is equal to $p$. The horizontal and vertical lines track the evolution of the expected fraction of erasure messages from the variable nodes to the check nodes at each iteration of the message-passing decoding algorithm.

Applying the stability condition and the equality $R=(1-\varepsilon) C$ in (9) gives

$$
C \varepsilon \geq a_{\mathrm{L}} p \lambda_{2} \sum_{i=0}^{l-1}\left|v_{i}\right|^{2} .
$$

Figure 1 implies that for an arbitrary iteration $l$

$$
1-\rho\left(1-x^{(l)}\right)=c\left(x^{(l)}\right)=1-\sum_{i=0}^{l}\left|v_{i}\right| .
$$

Substituting it in (5) yields that the average bit erasure prob. after decoding iteration number $l-1$ can be expressed as

$$
P_{\mathrm{b}}^{(l-1)}=p \cdot L\left(1-\sum_{i=0}^{l-1}\left|v_{i}\right|\right) .
$$

Let $l$ designate the number of iterations required to achieve an average bit erasure probability $P_{\mathrm{b}}$ over the ensembles, i.e., $l$ is the smallest integer which satisfies $P_{\mathrm{b}}^{(l-1)} \leq P_{\mathrm{b}}$ since we start counting at $l=0$. Although we consider an expectation over the LDPC ensemble, note that $l$ is deterministic as it is the smallest integer for which the average bit erasure probability does not exceed $P_{\mathrm{b}}$. Since $L$ is monotonically increasing, (11) provides a lower bound on $\sum_{i=0}^{l-1}\left|v_{i}\right|$ of the form

$$
\sum_{i=0}^{l-1}\left|v_{i}\right| \geq 1-L^{-1}\left(\frac{P_{\mathrm{b}}}{p}\right) .
$$

From the Cauchy-Schwartz inequality, we get

$$
\left(\sum_{i=0}^{l-1}\left|v_{i}\right|\right)^{2} \leq l \sum_{i=0}^{l-1}\left|v_{i}\right|^{2}
$$

Combining (10), (12) and (13), and solving for $l$ gives

$$
l \geq \frac{a_{\mathrm{L}} p \lambda_{2}\left(1-L^{-1}\left(\frac{P_{\mathrm{b}}}{p}\right)\right)^{2}}{(1-p) \varepsilon} .
$$

To continue the proof, we derive a lower bound on $1-L^{-1}(x)$ for $x \in(0,1)$. Since the fraction of variable nodes of degree $i$ is non-negative for all $i$, we have $L(x) \geq L_{2} x^{2}$ for $x \geq 0$. Substituting $t=L(x)$ and applying some algebra, we get

$$
1-L^{-1}(t) \geq 1-\sqrt{\frac{t}{L_{2}}}, \quad \forall t \in(0,1) .
$$

Under the assumption $\frac{P_{\mathrm{b}}}{p}<L_{2}$, substituting (15) in (14) gives

$$
l \geq \frac{a_{\mathrm{L}} \lambda_{2}\left(\sqrt{p L_{2}}-\sqrt{P_{\mathrm{b}}}\right)^{2}}{L_{2}(1-p) \varepsilon} .
$$

The lower bound in (1) is obtained by substituting the equality $L_{2}=\frac{\lambda_{2} a_{\mathrm{L}}}{2}$ into (16).

\section{B. Outline for the Proof of Theorem 3.2}

In [9, Section II.C.2], a technique called 'graph reduction' is introduced. This technique transforms the Tanner graph of a systematic ARA ensemble, transmitted over a BEC whose erasure probability is $p$, into a Tanner graph of an equivalent LDPC ensemble (where this equivalence holds in the asymptotic case where the block length tends to infinity). Let $(\widetilde{\lambda}, \widetilde{\rho})$ denote the degree distributions of the variable and parity-check nodes of the equivalent LDPC ensemble. The main observation used in the derivation of Theorem 3.2 is provided in the following lemma (for a proof, see [13]).

Lemma 4.2: Let $(n, \lambda, \rho)$ be an ensemble of systematic ARA codes whose transmission takes place over a BEC with erasure probability $p$. In the limit where the block length tends to infinity, the number of iterations required to achieve an average bit erasure probability $P_{\mathrm{b}}$ for the systematic bits is lower bounded by the number of iterations required to achieve an average bit erasure probability of $1-\sqrt{1-\frac{P_{b}}{p}}$ for the LDPC ensemble whose degree distributions are $\widetilde{\lambda}$ and $\widetilde{\rho}$. 
The LDPC ensemble with the tilted pair of degree distributions $(\widetilde{\lambda}, \widetilde{\rho})$ is transmitted over a BEC whose erasure probability is 1 , so the channel capacity is equal to zero and the multiplicative gap to capacity is meaningless. This prevents, unfortunately, a direct use of Theorem 3.1; however, the proof in [13] follows along the same lines as the proof of Theorem 3.1 with some necessary technical adjustments to relate between properties of the systematic ARA and the equivalent LDPC code ensembles.

\section{SUMmary AND CONCLUSIONS}

In this paper (for a full paper version, see [13]), we consider the number of iterations which are required for successful message-passing decoding of code ensembles defined on graphs. In the considered setting, we let the block length of these ensembles tend to infinity, and the transmission takes place over a binary erasure channel (BEC). For various families of code ensembles, Table I compares the number of iterations and the graphical complexity which are required to achieve a given fraction $1-\varepsilon$ (where $\varepsilon$ can be made arbitrarily small) of the capacity of a BEC with vanishing bit erasure probability. The results in Table I are based on lower bounds and some achievability results which are related to the graphical complexity of various families of code ensembles defined on graphs (see [8], [9], [11], [12]); the results related to the number of iterations are based on the lower bounds introduced here (for rigorous proofs, see [13]). Theorems 3.1-3.3 demonstrate that for various attractive

\begin{tabular}{|c|c|c|}
\hline $\begin{array}{c}\text { Code } \\
\text { family }\end{array}$ & $\begin{array}{c}\text { Number of iterations } \\
\text { as function of } \varepsilon\end{array}$ & $\begin{array}{c}\text { Graphical complexity } \\
\text { as function of } \varepsilon\end{array}$ \\
\hline \hline LDPC & $\Omega\left(\frac{1}{\varepsilon}\right)$ (Thm. 3.1) & $\Theta\left(\ln \frac{1}{\varepsilon}\right)[11]$ \\
\hline Systematic IRA & $\Omega\left(\frac{1}{\varepsilon}\right)$ (Thm. 3.3) & $\Theta\left(\ln \frac{1}{\varepsilon}\right)[12]$ \\
\hline Non-systematic IRA & $\Omega\left(\frac{1}{\varepsilon}\right)$ (Thm. 3.3) & $\Theta(1)[8]$ \\
\hline Systematic ARA & $\Omega\left(\frac{1}{\varepsilon}\right)$ (Thm. 3.2) & $\Theta(1)[9]$ \\
\hline
\end{tabular}

TABLE I

NUMBER OF ITERATIONS AND GRAPHICAL COMPLEXITY REQUIRED TO ACHIEVE A FRACTION $1-\varepsilon$ OF THE CAPACITY OF A BEC WITH VANISHING BIT ERASURE PROBABILITY.

families of code ensembles (including LDPC codes, systematic and non-systematic IRA codes, and ARA codes), the number of iterations which are required to achieve a fixed bit erasure probability scales at least like the inverse of the gap between the channel capacity and the design rate of the ensemble. This conclusion holds provided that the fraction of degree2 variable nodes in the Tanner graph does not vanish as the gap to capacity vanishes (where under mild conditions, this property is satisfied for sequences of capacity-achieving LDPC code ensembles, see [10, Lemma 5]). The behavior of these lower bounds matches well with the experimental results and the conjectures of Khandekar and McEliece [4].

\section{Acknowledgment}

This research work was supported by the Israel Science Foundation (grant no. 1070/07). This work was initiated during a visit of I. Sason at EPFL in Lausanne, Switzerland, and it benefited from a small unpublished write-up which was jointly written by S. Dusad, C. Measson, A. Montanari and R. Urbanke. This included preliminary steps towards the derivation of a lower bound on the number of iterations for LDPC code ensembles. I. Sason also wishes to acknowledge H. D. Pfister for discussions prior to this work on ARA codes.

\section{REFERENCES}

[1] A. Abbasfar, D. Divsalar, and K. Yao, "Accumulate-repeataccumulate codes," IEEE Trans. Commun., vol. 55, no. 4, pp. 692-702, April 2007.

[2] M. Ardakani, B. Smith, W. Yu and F. R. Kschischang, "Complexity-optimized low-density parity-check codes," Proceedings of the 43rd Annual Allerton Conference on Communication, Control and Computing, pp. 45-54, Urbana-Champaign, IL, USA, September 28-30, 2005.

[3] A. Ashikhmin, G. Kramer, and S. ten Brink, "Extrinsic information transfer functions: model and erasure channel properties," IEEE Trans. Inf. Theory, vol. 50, pp. 2657-2673, Nov. 2004.

[4] A. Khandekar and R. J. McEliece, "On the complexity of reliable communication on the erasure channel," Proceedings 2001 IEEE International Symposium on Information Theory, p. 1, Washington, D.C., USA, June 2001.

[5] M. G. Luby, M. Mitzenmacher, M. A. Shokrollahi and D. A. Spielman, "Efficient erasure-correcting codes," IEEE Trans. Inf. Theory, vol. 47, pp. 569-584, February 2001.

[6] X. Ma and E. Yang, "Low-density parity-check codes with fast decoding convergence speed," Proceedings 2004 International Symposium on Information Theory, p. 277, Chicago, IL, USA, June 2004.

[7] P. Oswald and A. Shokrollahi, "Capacity-achieving sequences for the erasure channel," IEEE Trans. Inf. Theory, vol. 48, pp. 3017-3028, December 2002.

[8] H. D. Pfister, I. Sason and R. Urbanke, "Capacity-achieving ensembles for the binary erasure channel with bounded complexity," IEEE Trans. Inf. Theory, vol. 51, pp. 2362-2379, July 2005.

[9] H. D. Pfister and I. Sason, "Accumulate-repeat-accumulate codes: Capacity-achieving ensembles of systematic codes for the erasure channel with bounded complexity," IEEE Trans. Inf. Theory, vol. 53, no. 6, pp. 2088-2115, June 2007.

[10] I. Sason, "On universal properties of capacity-approaching LDPC code ensembles," submitted to IEEE Trans. Inf. Theory, Sept. 2007.

[11] I. Sason and R. Urbanke, "Parity-check density versus performance of binary linear block codes over memoryless symmetric channels," IEEE Trans. Inf. Theory, vol. 49, pp. 1611-1635, July 2003.

[12] I. Sason and R. Urbanke, "Complexity versus performance of capacity-achieving irregular repeat-accumulate codes on the binary erasure channel," IEEE Trans. Inf. Theory, vol. 50, pp. 1247-1256, June 2004.

[13] I. Sason and G. Wiechman, "Bounds on the number of iterations for turbo-like ensembles over the binary erasure channel," submitted to IEEE Trans. Inf. Theory, November 2007. [Online]. Available: http://www.arxiv.org/abs/0711.1056.

[14] A. Shokrollahi, "Capacity-achieving sequences," IMA Volume in Mathematics and its Applications, vol. 123, pp. 153-166, 2000.

[15] S. ten Brink, "Convergence of iterative decoding," Electron. Lett., vol. 35, no. 10, pp. 806-808, May 1999. 